\title{
A Hierarchical Path Planning and Obstacle Avoidance System for an Autonomous Underwater Vehicle
}

\author{
F. Khorrami, P. Krishnamurthy
}

\begin{abstract}
In this paper, we propose a Maritime Underwater Navigation System (MUNS) for Unmanned Underwater Vehicles (UUVs). The proposed MUNS has a hierarchical architecture comprising of a Wide Area Planner (WAP) and a Local Area Planner (LAP). MUNS is intended to be a plug-and-play module to provide obstacle avoidance and path planning capabilities to existing UUV platforms thereby expanding operational capabilities in cluttered and littoral environments. MUNS is designed to be flexible and to support a variety of tasks including point-to-point moves, object inspection, contour tracking, and region scanning/searching. The performance of MUNS has been validated through extensive simulation studies.
\end{abstract}

\section{INTRODUCTION}

Unmanned Underwater Vehicles (UUVs) (and Unmanned Underwater Riverine Craft (UURC)) can play an important role in many civilian and military applications [1-3] such as intelligence and surveillance applications, search and rescue, mobile communication relays, and hull and pier inspection with object identification and localization. To realize their full potential in these and other applications, it would be greatly beneficial to attain a high degree of autonomy, reliability, and robustness in order to negotiate unknown hazardous environments, and to perform a launch-and-forget mission in which the vehicle travels to the target point without operator intervention. Achieving autonomy capabilities in UUVs, especially in riverine, harbor, shallow water, or cluttered environments forms a challenging problem due to various sensory limitations [4-11]. Sonar sensors which form the primary sensor modality for picking up information on local underwater geometry are noisy, affected by surface returns and water turbulence, and offer only a limited field of view. Furthermore, in typical UUV applications, a priori information on the local obstacle geometry could be minimal or nil thus making the obstacle avoidance task completely dependent on real-time sensor information. Additionally, in underwater applications, position referencing sonar returns into an absolute frame could be difficult since the estimate of the vehicle's own position drifts in the absence of GPS updates (this is particularly relevant when it is desirable for stealth reasons that the UUV not surface for GPS fixes). Hence, the obstacle avoidance module should function reliably with purely relative measurements even when the vehicle's own position is uncertain.

In this paper, we describe the Maritime Underwater Navigation System (MUNS), a path planning and obstacle avoidance system intended to be a plug-and-play module for UUVs and UURC. The design of MUNS is based on our earlier work on a low-resource algorithm GODZILA (Game-Theoretic Optimal Deformable Zone with Inertia and Local Approach) [12,13] and its application along with the $A^{*}$ search algorithm $[14,15]$ to attain a hierarchical path planning and obstacle avoidance system for Unmanned Surface Vehicles (USVs) [16,17].

The first author is with Control/Robotics Research Laboratory (CRRL), Department of Electrical and Computer Engineering, Polytechnic University, Brooklyn, NY, 11201 and with IntelliTech Microsystems, Inc. (IMI), Bowie, MD, 20715. The second author is with IMI.

This work was supported in part by DARPA under contract no. W31P4Q08-C-0058.

Approved for Public Release, Distribution Unlimited.

Corresponding Author: F. Khorrami, khorramiasmart.poly.edu

\section{MUNS AND ITS COMPONENTS}

The MUNS module (Figure 1) described in this paper addresses the path planning and obstacle avoidance problem for a UUV operating in partially or completely unknown environments through the utilization of a hierarchical path planning and obstacle avoidance system comprising of a Wide Area Planner (WAP) and a Local Area Planner (LAP). The WAP is responsible for higher-level planning given the mission specifications while the LAP focuses on local obstacle avoidance. The LAP can operate based on just the relative measurements to obstacles provided by the sonars at the current time while the WAP constructs and maintains an environment map to achieve the specified mission objective. The utilization of a modular hierarchical approach makes it straightforward to address different mission objectives (including collaborative missions performed by multiple unmanned vehicles) through inclusion of appropriate algorithms in the WAP component. In this effort, the LAP is based on GODZILA which is an optimization-based reactive obstacle avoidance algorithm.

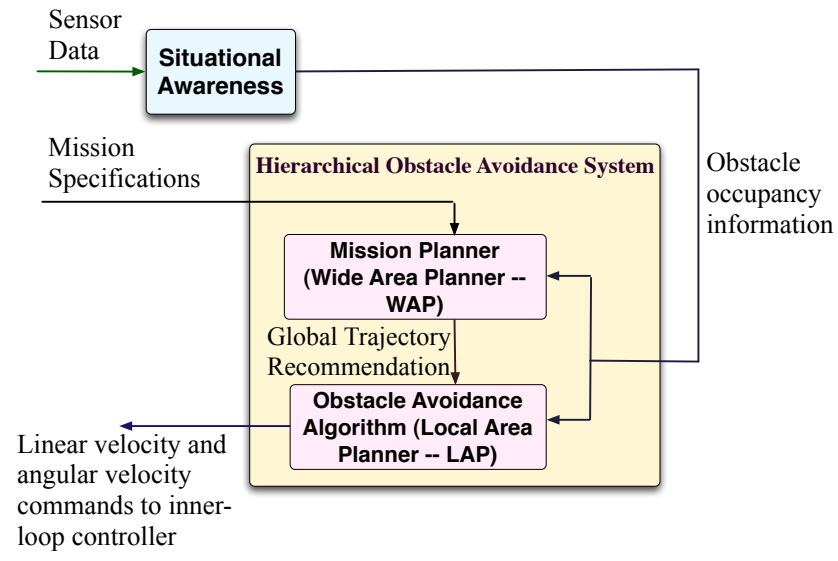

Fig. 1. Conceptual architecture of overall MUNS.

MUNS includes three central components: (1) Situational Awareness: The data from the sensors is integrated into a coherent knowledge base of the obstacle geometry taking into account uncertainties resulting from sensor noise and drift in own position estimate. (2) WAP: The WAP is a higherlevel module (Mission Planner) which addresses the specific mission (such as searching a specified region, examining a specific object by tracing its contour, or visiting a sequence of waypoints) being performed and computes nominal UUV trajectories to execute the mission. (3) LAP: The LAP is responsible for local obstacle avoidance, i.e., maintaining the specified safe clearance distance to the obstacles in the vicinity. To accomplish this task, the LAP modifies in realtime the nominal trajectory computed by the WAP. The WAP typically operates using a coarse map over a larger geographical region and updates at a much lower sampling rate than the obstacle avoidance algorithm. For simple pointto-point moves, the WAP is not required and the LAP can operate on its own given only the final target position.

The overall OAS which is comprised of the situational 
awareness component, the LAP, and the WAP is responsible for translating given task specifications into linear and angular velocity commands to the inner-loop controller (autopilot), which in turn computes the physical actuator commands to track the commanded linear and angular velocities. The UUV is equipped with acoustic sensors (sonars) to detect obstacles in the environment. The sensor readings from each sonar (after preliminary processing) yields a grid of measurements of range to obstacles from which the range measurements utilized by GODZILA are computed.

\section{A. Situational Awareness}

The sensor data from sonars are processed by the situational awareness block to obtain a coherent obstacle map of the environment taking into account the sensor noise and uncertainty in the UUV's position. The construction of the obstacle map by the situational awareness block involves a probabilistic characterization of the obstacle geometry (modeled through a set of probabilistic occupancy grids), propagation of the sensor data over time based on estimates of the UUV's motion, and examination of the obstacle detection persistence over time. Depending on the particular task being performed, the situational awareness block also keeps track of additional data such as the history of the UUV's position (this information is useful in a region scanning or searching mission). If additional sensory or geographic information is available such as turbulence locations/patterns, then the situational awareness block can also integrate such information into occupancy grids for use by the WAP to assign traversal costs to cells in the grid. The situational awareness block provides easily adjustable tuning parameters such as grid sizes and resolutions, smoothing function for entering sonar return information into the grids, forgetting factors to control persistence of information in the grids, etc.

\section{B. WAP Designs}

The WAP includes a suite of algorithms for common tasks. The MUNS is designed in a modular fashion to easily allow incorporation of additional algorithms in WAP to extend functionality to different types of missions. Currently, the WAP is a combination of $A^{*}$, heuristics, and dynamic optimization. The WAP accepts high-level mission objectives and computes nominal desired motion direction. The WAP also deforms the local topology as seen by GODZILA to attain desired behavior from GODZILA. WAP includes heuristics and dynamic optimization to address specifications such as keep-inside region (i.e., operating region), keep-out region, minimum clearance to obstacles, etc. In the simplest case of a point-to-point move, the WAP performs an $A^{*}$ search $[14,15]$ to compute the optimal path from the current position to the target location based on currently available information as obtained from the situational awareness block. A discrete grid is used for this purpose with traversal costs of the cells in the grid propagated by spatial averaging and smoothing from the probabilistic occupancy grids maintained by the situational awareness block. Criteria considered in the computation of the traversal cost include nearness to obstacles, depth, and vehicle kinematic constraints. In addition, for tasks such as region scanning, distance of a cell from the position history grid (used by the situational awareness block to keep track of locations visited by the vehicle) is also considered in computation of the cell traversal cost. The cost function for optimization in $A^{*}$ is the total path length (taking kinematic constraints into account). If additional sensory or geographic information is available such as turbulence locations/patterns, then such information can be readily integrated into the search by modifying traversal costs of cells appropriately to encourage finding of paths that avoid regions of turbulence. Similarly, if a specific behavior is required in a mission scenario such as requiring the UUV to keep near the middle of a river, then such behavior can be attained in a straightforward way by modifying the cell traversal costs. To provide a high-level interface to easily control such behaviors, the WAP has been designed to accept commands from a higher level block as to desired and/or undesired regions or criteria, the intent being that a higher level mission planner will determine required behavior and model that required behavior in terms of a set of criteria to input to the WAP.

\section{Application of GODZILA for LAP Design}

The UUV OAS is designed based on GODZILA [12] which is a versatile general-purpose computationally lightweight algorithm for obstacle avoidance for various types of unmanned vehicles. GODZILA does not require any a priori information about the environment and does not rely on building an obstacle map. GODZILA works in any finite-dimensional space with provable convergence with probability 1 to target. The algorithm follows a purely local approach using only the sensor measurements at the current time and requiring only a small number of stored variables in memory. This minimizes the memory and computational requirements for implementation of the algorithm, a feature that is especially attractive for small autonomous vehicles. The trajectory is generated through the online solution of an optimization cost at each sampling instant. It can be shown that the optimization cost can be chosen so that the minimizer can be obtained in closed form. The optimization cost includes three terms which penalize, respectively, motion in directions other than the direction to the target, motion towards obstacles, and back-tracking. In addition to the optimization algorithm, GODZILA includes two components, a local straight-line planner utilized if the target is visible and navigation towards a random target. Since the algorithm follows a local approach, it is possible to be caught in a local minimum. When a local minimum or a "trap" is detected, navigation towards a random target is initiated to escape the trap. For enhanced efficacy in UUV applications, the various design freedoms (parameters and cost functions) appearing in the GODZILA algorithm have been fine-tuned for UUV applications based on simulations incorporating the kinematic and dynamic characteristics of the UUV, the sensor characteristics, and the typical operating conditions. In addition, the GODZILA algorithm has been customized in multiple directions as outlined below:

1) In littoral environments, the UUV would need to operate in close proximity to the seafloor. Hence, keep-out distance tolerance for the vertical direction would need to be smaller than for the horizontal plane. However, since the seafloor is not of constant depth (there is also clutter and debris on the seafloor), the OAS should be sensitive to depth variations. These considerations are addressed in the GODZILA framework by generalizing the optimization cost function component $J_{2}$ in GODZILA (which penalizes motion towards obstacles - see [12]) to include a depth-modulated directional weighting wherein sensor returns indicating $z$ axis (vertical) proximity to obstacles appear into the obstacle avoidance component of optimization cost with low clearance requirement but with high cost growth at close proximities. 
2) To utilize the full range of maneuvering capabilities of the UUV, the optimization component of GODZILA is enhanced to compute both optimal yaw orientation $\left(\theta_{y}\right)$ and optimal direction of motion. The optimal direction of motion is computed as in the standard GODZILA algorithm with the modification described above and the optimal yaw orientation is computed by minimizing an optimization cost of the form $J_{y}(y)=$ $J_{s y}\left(\Delta_{s}\left(\theta_{y}\right)\right)+J_{h}\left(\theta_{y}\right)+J_{t}\left(\theta_{y}\right)$ where

- $J_{s y}$ is a class- $\mathcal{K}$ function of $\Delta_{s}\left(\theta_{y}\right)$ where $\Delta_{s}\left(\theta_{y}\right)$ is defined as the amount of yaw required to bring the direction in which the closest sensor reading is currently being detected into the center of the field of view of any one of the sensors mounted on the vehicle, i.e., the action of the component $J_{s y}$ is to encourage the UUV to align itself so that the direction in which obstacle proximity is closest is within the field of view of one of the sensors.

- $J_{h}\left(\theta_{y}\right)$ penalizes the deviation between the horizontal plane vector corresponding to the yaw command and the current linear velocity vector projected into the horizontal plane.

- $J_{t}\left(\theta_{y}\right)$ penalizes the deviation between the horizontal plane vector corresponding to the yaw command and the vector to the desired target location projected into the horizontal plane.

MUNS maintains dual frame (absolute/relative) situational awareness and the OAS can function purely with relative distances to obstacles thus providing robustness to navigation drift. Therefore, MUNS does not introduce any additional vehicle position accuracy requirements beyond what is needed in terms of mission specifications (e.g., if the vehicle is commanded to go to a point within accuracy of $10 \mathrm{~m}$, then the position drift of the navigation system should not accumulate to more than $10 \mathrm{~m}$ minus the maneuvering accuracy of the UUV; similarly, for a region scanning mission, the coverage distance and the allowed excursion beyond the operational area set the required position accuracy). The position accuracy demanded by mission specifications governs how often the vehicle would need to surface for GPS updates. The operational endurance and range are dictated by vehicle specifications (e.g., fuel, batteries, communication range if real-time communication is desired, storage capacity if data logging is desired, etc.) and are not further limited by MUNS except for the power penalty for introduction of additional sonars if required; MUNS can operate with a single forward-looking sonar as seen in the simulation studies in Section IV although utilization of four sonars (one pointing forward, two pointing to the two sides, and one pointing downward) would be recommended if the vehicle's SWaP (size, weight, and power) constraints allow it.

\section{Applications to Contour Tracking AND REGION SEARCH Missions}

To address common tasks such as tracking/examining the contour of a specified object and scanning/searching a specified region, WAP algorithms have been developed and integrated into the WAP module. The conceptual architectures of the overall system when operating in these modes are illustrated in Figures 2 and 3, respectively. In each of these two modes, the WAP includes an algorithm to decide on the "next location" to visit (which we will refer to as a pseudo-target) based on the task requirements; this location then forms the input to the point-to-point mode WAP/LAP OAS (i.e., $A^{*}$ in conjunction with GODZILA).

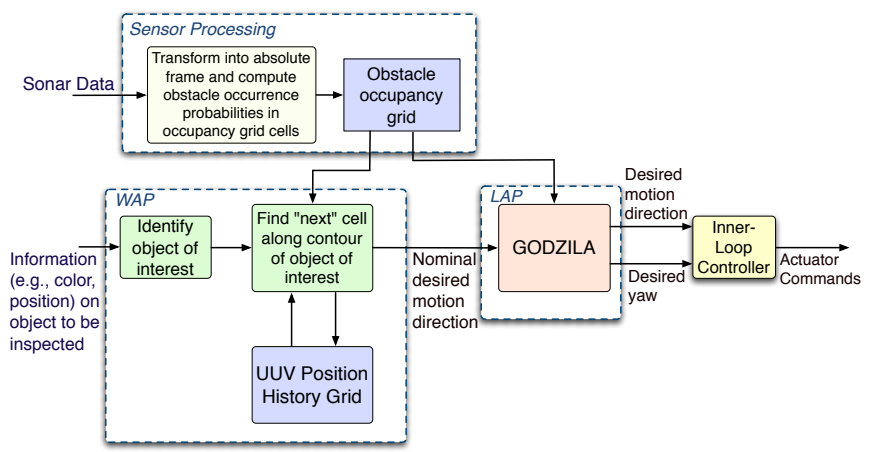

Fig. 2. MUNS: Application to contour tracking.

In the case of contour tracking, the pseudo-target is picked to be the estimate of the "next" cell along the contour of the object of interest; the properties used to characterize this next cell include a lower bound on distance from locations traversed within a small past time window, bound (both lower and upper - based on the desired tracking distance) on distance from the boundary of the object, and upper bound on difference in angle between current heading and vector from current location to the next cell. It is evident that the characteristics defining the next cell allow several tunable parameters. Similarly, in region scanning, the pseudo-target is picked along the "next" scan line, which is characterized based on a lower bound on distance from previously visited locations and a lower bound on distance from obstacles. As mentioned above, a path to the current pseudo-target is determined using the $A^{*}$ and GODZILA algorithms. In both the contour tracking and the region scanning cases, picking of the pseudo-target is based on a breadth-first search for the nearest cell possessing the aforementioned properties. MUNS has been designed to seamlessly switch between different modes during run time.

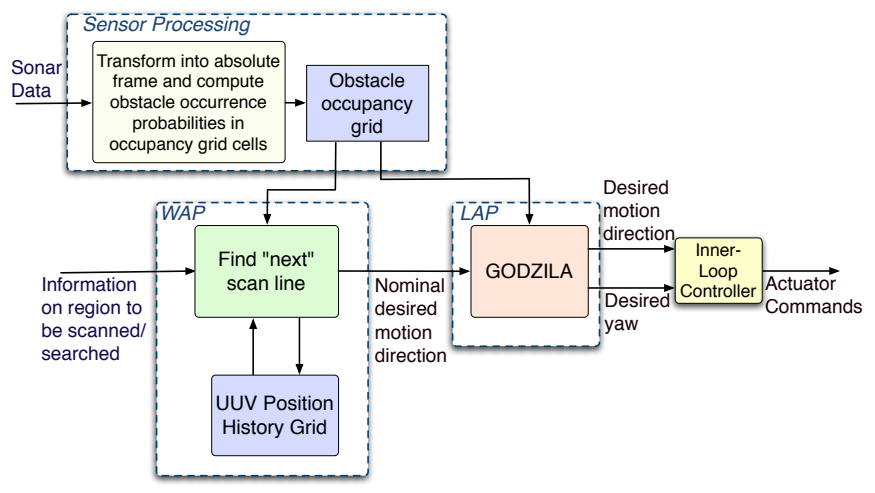

Fig. 3. MUNS: Application to region scanning.

\section{Simulation Studies}

\section{A. Simulation Platform}

A dynamic simulation platform with the architecture shown in Figure 4 has been constructed incorporating the UUV operating characteristics, an environment model, sonar model, and the GODZILA OAS. The simulation platform supports an arbitrary number of static and moving obstacles in the environment. The obstacle geometry is specified at runtime through text configuration files. The simulator has been implemented in the $\mathrm{C}++$ programming language and includes a 3D OpenGL based graphical user interface for ease in visualization and demonstration of the OAS performance. To allow application of the simulator to future work on collaborative planning and obstacle avoidance for multiple UUVs, the simulator has been designed from the ground up 
to include support for an arbitrary number of UUVs (with possibly different dynamic and kinematic characteristics).

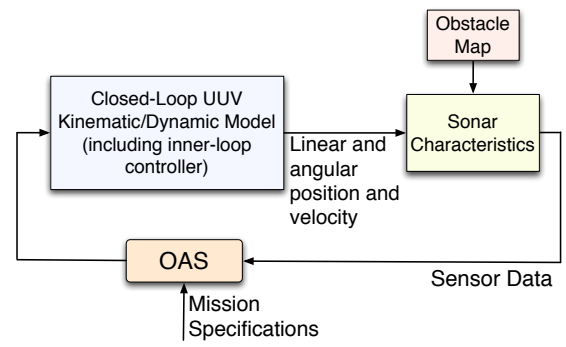

Fig. 4. Architecture of the simulation platform.

\section{B. Modeling of UUV and Sensors}

The simulation platform supports inclusion of both kinematic motion constraints and also a dynamic model of the UUV motion. The motion constraints addressed in the simulation studies outlined in Section IV-C include maximum speed (horizontal, i.e., at constant depth) of $2 \mathrm{~m} / \mathrm{s}$, maximum acceleration of $0.2 \mathrm{~m} / \mathrm{s}^{2}$, maximum deceleration of $0.35 \mathrm{~m} / \mathrm{s}^{2}$, maximum dive/climb speed of $0.3 \mathrm{~m} / \mathrm{s}$, maximum turn rate of $10 \mathrm{deg} / \mathrm{s}$, and minimum turn radius of $10 \mathrm{~m}$.

As shown in Figure 4, the output of the closed-loop kinematic/dynamic model of the UUV provides linear and angular position and velocity signals which are then used to synthesize a simulation of sonar data as a grid of range measurements. The number of measurements in the grid for each sonar is determined by the sonar's field of view and its angular resolution. If the horizontal field of view is denoted by $\mathrm{FOV}_{h}$ (i.e., the horizontal field of view is $\left.\left[-\frac{\mathrm{FOV}_{h}}{2}, \frac{\mathrm{FOV}_{h}}{2}\right]\right)$ and the vertical field of view is denoted by $\mathrm{FOV}_{v}$ (i.e., the vertical field of view is $\left[-\frac{\mathrm{FOV}_{v}}{2}, \frac{\mathrm{FOV}_{v}}{2}\right]$ ), then the sonar measurements are represented by a matrix of dimension $\left(2 N_{h}+1\right) \times\left(2 N_{v}+1\right)$ where $N_{h}=\frac{\mathrm{FOV}_{h}}{2 \delta \theta_{h}}$ and $N_{v}=\frac{\mathrm{FOV}_{v}}{2 \delta \theta_{v}}$ with $\delta \theta_{h}$ and $\delta \theta_{v}$ denoting the horizontal and vertical angular resolutions, respectively, of the sonar. Given an obstacle set $u_{e}$ expressed in inertial frame, the sonar measurements of a sonar mounted with axis vector $a_{s}$ represented in body frame (body frame axes are constructed with $X$ axis pointed towards the front of the vehicle, $Y$ axis to the left, and $Z$ axis downwards) are ideally given by

$$
\begin{aligned}
& s_{(i, j)}=\min \left\{d \mid x_{p}+d\left(x_{h}+q_{(i, j)}\right) \in u_{e}\right\} \\
& \quad \text { for } i \in\left\{-N_{h}, \ldots, N_{h}\right\}, j \in\left\{-N_{v}, \ldots, N_{v}\right\}
\end{aligned}
$$

where $x_{p}$ is the current position, $x_{h}$ is the unit vector along the current heading (expressed in inertial frame, i.e., if the rotation matrix between the body frame and the inertial frame in the current orientation is $R_{i}^{b}$, then $x_{h}=R_{i}^{b}[1,0,0]^{T}$ ), and

$$
q_{(i, j)}=R_{i}^{b}\left(R_{y, j \delta \theta_{v}} R_{z, i \delta \theta_{h}} a_{s}-[1,0,0]^{T}\right) .
$$

with $R_{y, \theta}$ denoting the rotation matrix corresponding to a counter-clockwise rotation by angle $\theta$ around the $Y$ axis and $R_{z, \theta}$ denoting the rotation matrix corresponding to a counterclockwise rotation by angle $\theta$ around the $Z$ axis. Physically, the ideal measurements given by (1) are corrupted by three factors, namely sensor range $(R)$, sensor linear resolution $\left(r_{l}\right)$, and sensor angular resolution $\left(r_{a}\right)$, resulting in the replacement of (1) by

$$
\begin{aligned}
s_{(i, j)}= & \operatorname{sat}_{R} \min \left\{d \mid x_{p}+d\left(x_{h}+\tilde{q}_{(i, j)}\right) \in u_{e}\right\}+\tilde{s}_{(i, j)}, \\
& i \in\left\{-N_{h}, \ldots, N_{h}\right\}, j \in\left\{-N_{v}, \ldots, N_{v}\right\}
\end{aligned}
$$

where $\tilde{s}_{(i, j)}$ and the deviation of $\tilde{q}_{(i, j)}$ from $q_{(i, j)}$ are governed by random distributions (nominally modeled as zero-mean Gaussian for the purpose of simulation with standard deviations $r_{l}$ and $r_{a}$, respectively) and sat ${ }_{R}$ denotes a saturation to a maximum of $R$.

\section{Simulation Results}

The proposed MUNS module has been validated through extensive simulations using the dynamic simulation platform illustrated in Figure 4 that incorporates the UUV operating characteristics and a model of the environment and sensors. Simulations have been performed with a wide range of topologies typically encountered in UUV applications. Simulation studies have also been carried out with various numbers and placements of sensors and various levels of sensor performance to estimate the OAS performance that can be attained in different scenarios. Representative simulation results are briefly summarized in this section.

Screenshots for sample simulation runs for point-to-point navigation are shown in Figures 5 and 6 . In each of the screenshots, the red sphere indicates the UUV's initial location while the green sphere indicates the target location. The inputs to the WAP/LAP OAS are range measurements corresponding to four acoustic sensors (one pointing forward, two pointing to the two sides, and one pointing downward) with range $50 \mathrm{~m}$, linear resolution 6 inches, angular resolution 0.5 degree, and field of view 25 degrees. The simulated trajectory of the UUV under the online action of the WAP/LAP OAS is highlighted by the yellow cuboids. In Figure 5, the environment contains nine obstacles (consisting of five cuboids, one of which is completely submerged, and four mine-like spheres tethered to the seafloor) in addition to the seafloor. The geometry in Figure 6(Top) requires the UUV to find its way out of an enclosed area while in Figure 6(Bottom), the UUV needs to find its way out of an enclosed area and then navigate around an obstacle across the exit of the enclosed area. As mentioned earlier, the simulations in Figures 5 and 6 are with four sensors. To evaluate the performance when only one sensor is utilized, simulations were performed as shown in Figure 7. It is noted that MUNS is indeed able to function reliably with a single sensor; however, performance is noticeably degraded especially in Test Case 2 showing increased wandering of the UUV. In Figure 8, screenshots are shown for simulations wherein a position drift (i.e., error in estimate of the vehicle's own position) is included with error in estimate of the vehicle's own position given by $(10+0.005 t) \mathrm{m}$ in both $\mathrm{X}$ and $\mathrm{Y}$ axes (this high rate of drift is used here to illustrate, within a short simulation run, the OAS behavior under position drift). It is seen that this position uncertainty results in error in the final stopping location of the UUV (i.e., shift from the actual target location); however, the obstacle avoidance itself is unaffected by position uncertainty since the LAP operation is purely in relative frame and does not rely on knowledge of absolute position of the UUV. This is a desirable feature since UUV position estimate does drift over time when the UUV does not surface for GPS updates. To examine performance deterioration under higher sensor noise, a simulation was run with double the sensor noise (i.e., 1 foot sensor accuracy) in Figure 9 and it is seen that performance remains adequate thus building confidence that the proposed system would be able to operate even under adverse environmental and turbulence conditions wherein sensor accuracy could be degraded.

Sample simulation runs for contour tracking are illustrated in Figure 10 for three simulation scenarios: (a) With four 
sensors as discussed above (b) With only one forward looking sensor (c) With four sensors but with uncertainty in the vehicle's own position (position drift of $(10+0.005 t) \mathrm{m}$ in both $\mathrm{X}$ and $\mathrm{Y}$ axes). In each of the simulation runs, the objective was to track the (a priori unknown) contour of the obstacle with a desired stand-off distance of $10 \mathrm{~m}$. The red circle denotes the starting location of the UUV. Note that at initialization time, the algorithm has no knowledge of the obstacle contour and the only piece of identifying information provided is the coordinate location of a single point inside the object of interest. The distances from the obstacle as a function of time during the contour tracking operations are shown in Figure 11 for four simulation scenarios (four sensors as discussed above, only one forward looking sensor, four sensors but with drift in own position estimate, one sensor with drift in own position estimate). It is seen that when four sensors are used, the performance is quite robust to uncertainty in the vehicle's own position. The performance with a single forward looking sensor shows more variation in stand-off distance, but could be acceptable given the task requirements.

Simulations for a sample simulation run for region scanning are shown in Figure 12. The region of interest is specified through a sequence of coordinate locations forming the vertices of a polygon. Simulations were also performed to evaluate the effect of a drift in the UUV's own position estimate. As expected, it was seen that a drift in the vehicle's own position results in a shift in the perceived region of interest. It is conceivable that in a scenario such as this, the effects of position drift could be alleviated by monitoring the obstacle geometry over time and using mismatch between a recorded map and currently observed geometry to compensate for the drift in the vehicle's own position estimate; this is more feasible if some obstacle is known to be fixed and can be used as a beacon). While such drift correction using obstacle geometry is not further pursued in this effort, it is understood that an algorithm (along with appropriate sensors and processing) to perform such a position drift estimation can be incorporated into the entire system to provide a compensating effect against drift thus improving OAS performance for tasks such as region scanning or pointto-point moves wherein absolute frame position knowledge is required to meet task specifications.
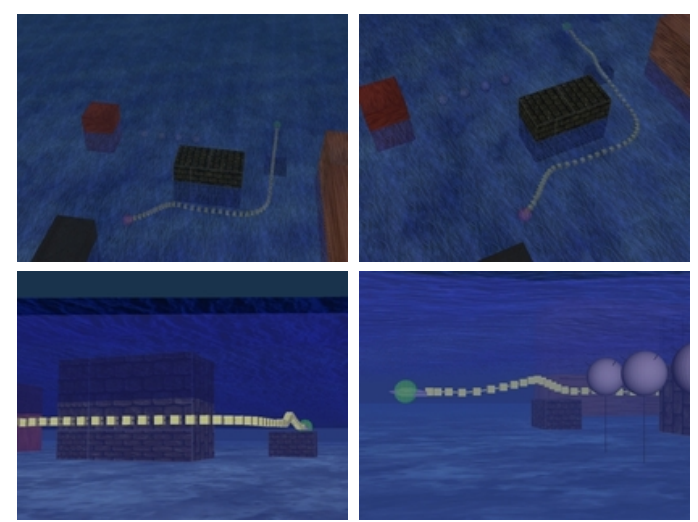

Fig. 5. UUV simulation with four sensors for Point-to-Point Test Case 1.

\section{CONCLUSION}

In this paper, we have described MUNS, a hierarchical scalable real-time dynamic OAS and path planning module. MUNS has been designed to be computationally lightweight, reliable, and capable of straightforward plugand-play integration into existing UUV systems. The MUNS
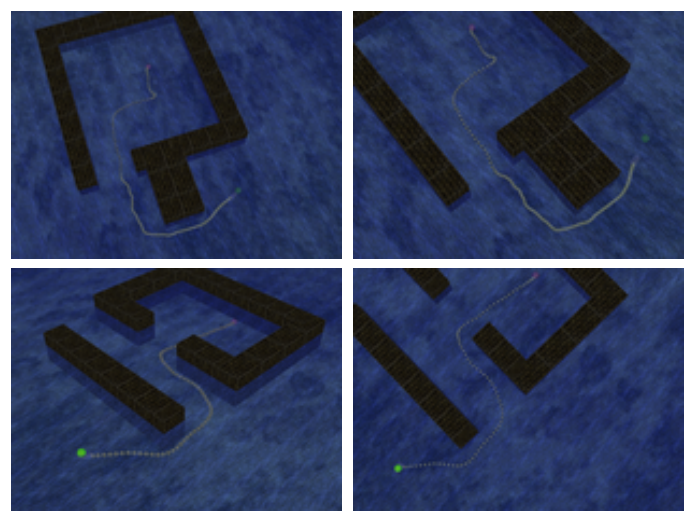

Fig. 6. UUV simulation with four sensors: Point-to-Point Test Cases 2 (Top) and 3 (Bottom).
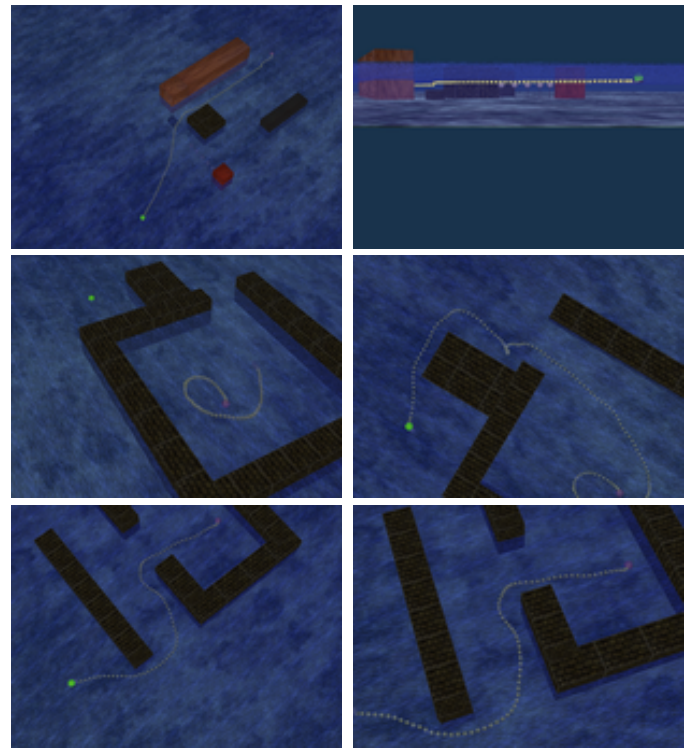

Fig. 7. Sample UUV simulation runs with one forward-looking sensor: Point-to-Point Test Cases 1 (Top), 2 (Middle), and 3 (Bottom).
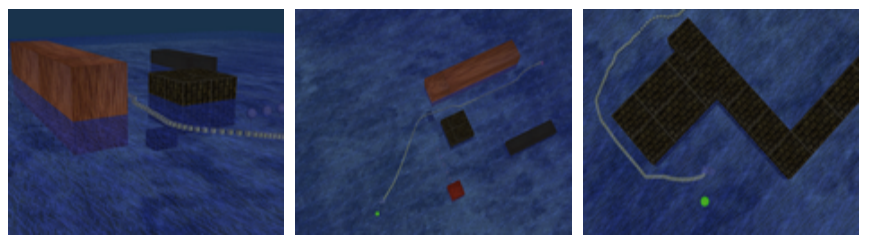

Fig. 8. Sample UUV simulation runs with four sensors and drift in position estimate: Point-to-Point Test Cases 1 (Left and Middle) and 2 (Right).
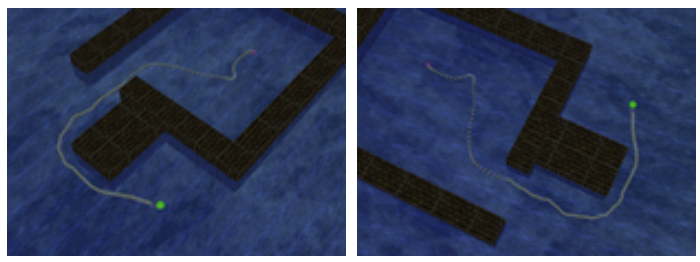

Fig. 9. Sample UUV simulation run with four sensors and higher sensor noise: Point-to-Point Test Case 2.

is designed to be integrated onto existing UUV platforms in either of the following two configurations: firstly, as a plug-and-play stand-alone module communicating with an existing UUV autopilot via a message-based JAUS-compliant communication interface, and secondly, as a software module incorporated into an existing processing module on the UUV. The modular nature of MUNS also allows benefiting from 

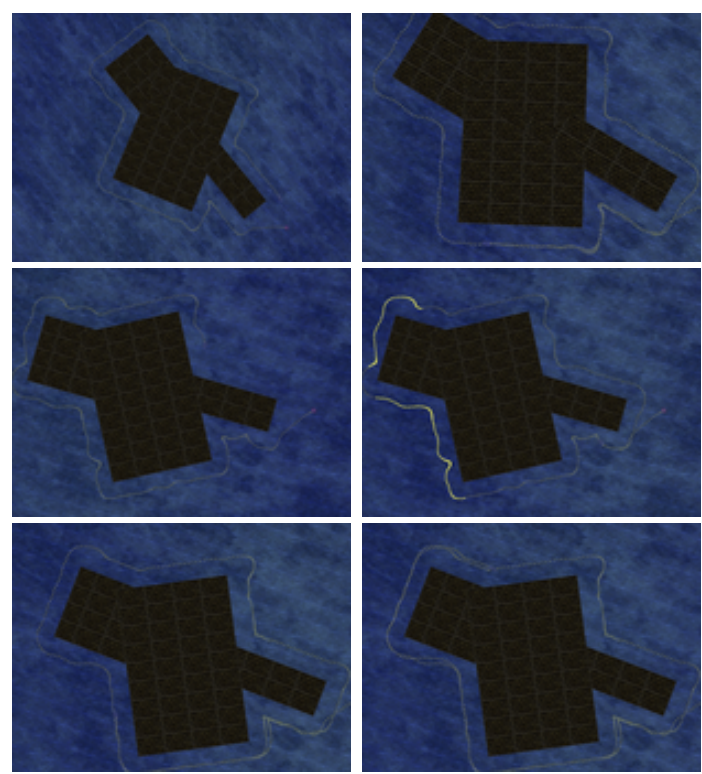

Fig. 10. Sample UUV simulation runs for contour tracking: top two screenshots are with four sensors; middle two screenshots are with one forward-looking sensor; bottom two screenshots are with four sensors and drift in position estimate.

other existing modules which provide functionality such as computing movement recommendations based on energy harvesting or turbulence considerations.

\section{REFERENCES}

[1] B. Fletcher, "UUV master plan: a vision for navy UUV development," in Proc. of the OCEANS 2000 MTS/IEEE Conference and Exhibition, Providence, RI, Sept. 2000, pp. 65-71.

[2] G. Griffiths, Technology and Applications of Autonomous Underwater Vehicles. CRC Press, 2002.

[3] J. D. Lambert, P. Picarello, and J. E. Manley, "Development of UUV standards, an emerging trend," in Proc. of the OCEANS 2006 MTS/IEEE Conference and Exhibition, Boston, MA, Sept. 2006, pp. $1-5$.

[4] E. O. Belcher, W. L. J. Fox, and W. H. Hanot, "Dual-frequency acoustic camera: a candidate for an obstacle avoidance, gap-filler, and identification sensor for untethered underwater vehicles," in Proc. of the OCEANS 2002 MTS/IEEE Conference and Exhibition, Biloxi, MS, Oct. 2002, pp. 2124-2128.

[5] M. P. Olivieri, "Bio-inspired broadband SONAR technology for small UUVs," in Proc. of the OCEANS 2002 MTS/IEEE Conference and Exhibition, Biloxi, MS, Oct. 2002, pp. 2135-2144.

[6] C. D. Loggins, "A comparison of forward-looking sonar design alternatives," in Proc. of the OCEANS 2001 MTS/IEEE Conference and Exhibition, Honolulu, HI, Nov. 2001, pp. 1536-1545.

[7] I. T. Ruiz, S. de Raucourt, Y. Petillot, and D. M. Lane, "Concurrent mapping and localization using sidescan sonar," IEEE Journal of Oceanic Engineering, vol. 29, no. 2, pp. 442-456, Apr. 2004.

[8] L. C. Langebrake, S. A. Samson, E. A. Kaltenbacher, E. T. Steimle, J. T. Patten, C. E. Lemble, R. H. Byrne, and K. Carder, "Sensor development: progress towards systems for AUVs/UUVs MTS Oceans 2000," in Proc. of the OCEANS 2000 MTS/IEEE Conference and Exhibition, Providence, RI, Sept. 2000, pp. 617-621.

[9] B. Kalyan and A. P. Balasuriya, "Multisensor data fusion approach for terrain aided navigation of autonomous underwater vehicles," in Proc. of the OCEANS 2004 MTTS/IEEE TECHNO-OCEAN, Kobe, Japan, Nov. 2004, pp. 2013-2018.

[10] T. Nicosevici, R. Garcia, M. Carreras, and M. Villanueva, "A review of sensor fusion techniques for underwater vehicle navigation," in Proc. of the OCEANS 2004 MTTS/IEEE TECHNO-OCEAN, Kobe, Japan, Nov. 2004, pp. 1600-1605.

[11] P. Bouxsein, E. An, S. Schock, and P.-P. Beaujean, "A SONAR simulation used to develop an obstacle avoidance system," in Proc. of the OCEANS 2006 Asia Pacific, Singapore, May 2006, pp. 1-7.

[12] P. Krishnamurthy and F. Khorrami, "GODZILA: A low-resource algorithm for path planning in unknown environments," in Proc. of the American Control Conference, Portland, OR, June 2005, pp. 110115 , also in the Journal of Intelligent and Robotic Systems, vol. 48, no. 3, pp. 357-356, March 2007.
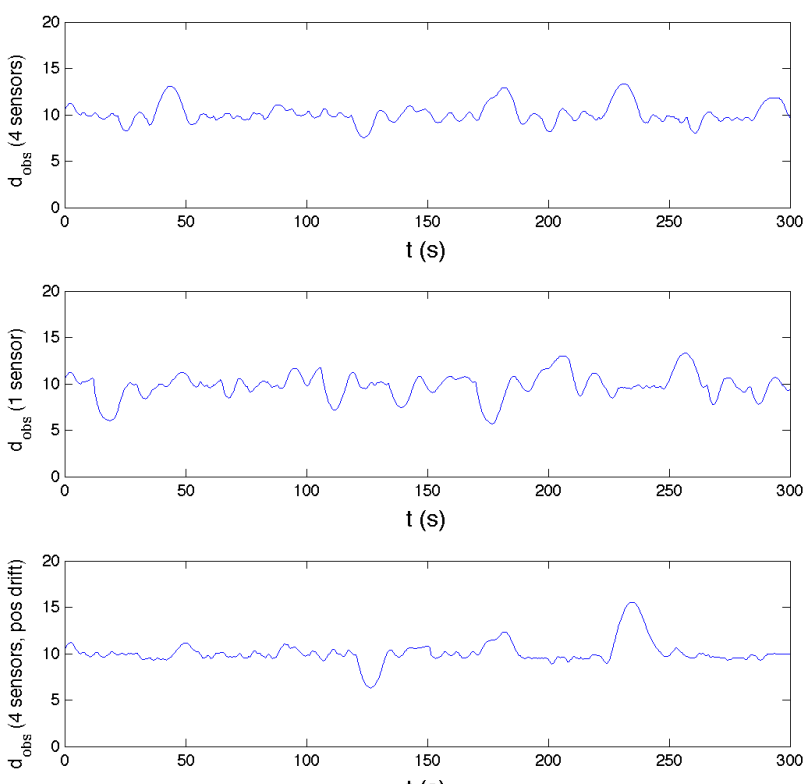

$t(s)$

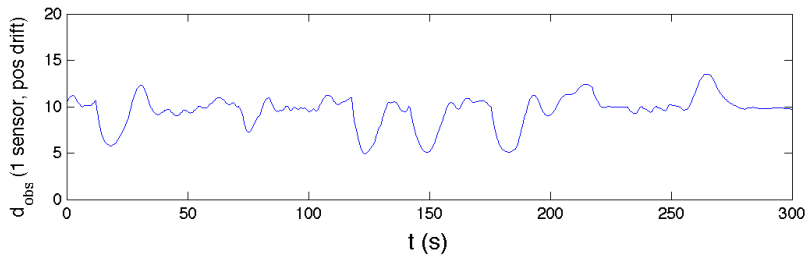

Fig. 11. Distance from obstacle for sample UUV simulation runs for contour tracking.
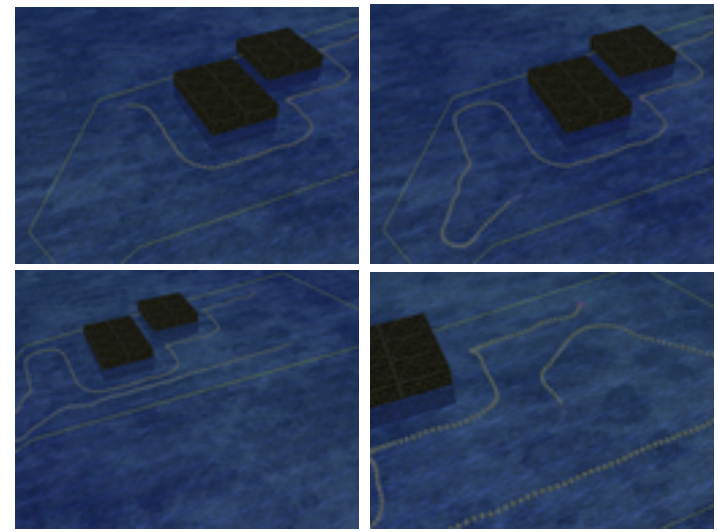

Fig. 12. Sample UUV simulation with four sensors: Region Scanning.

[13] T. L. Ng, P. Krishnamurthy, F. Khorrami, and S. Fujikawa, "Autonomous flight control and hardware-in-the-loop simulator for a small helicopter," in Proc. of the 2005 IFAC World Congress, Prague, Czech Republic, July 2005.

[14] P. E. Hart, N. J. Nilsson, and B. Raphael, "A formal basis for the heuristic determination of minimum cost paths," IEEE Trans. on Systems Science and Cybernetics, vol. SSC-4, no. 2, pp. 100-107, July 1968.

[15] R. Dechter and J. Pearl, "Generalized best-first search strategies and the optimality of $A^{*}$, , Journal of the ACM, vol. 32, no. 3, pp. 505-536, July 1985.

[16] P. Krishnamurthy, F. Khorrami, and T. L. Ng, "Control design for unmanned sea surface vehicles: Hardware-in-the-loop simulator and experimental results," in Proc. of the 2007 International Conference on Intelligent Robots and Systems, San Diego, CA, Oct. 2007.

[17] _ - "Obstacle avoidance for unmanned sea surface vehicles: a hierarchical approach," in Proc. of the 2008 IFAC World Congress, Seoul, Korea, July 2008. 